\title{
SOLAR SOFT X-RAYS AND SOLAR ACTIVITY
}

\section{Observational Assessment of the Role of the Type III Acceleration Mechanism in Establishment of the Soft X-Ray Source Volume}

\author{
RICHARD G. TESKE \\ Department of Astronomy and the McMath-Hulbert Observatory, \\ The University of Michigan, Ann Arbor, Mich., U.S.A. \\ and \\ ROGER J. THOMAS \\ Laboratory for Solar Physics, NASA/Goddard Space Flight Center, Greenbelt, Md., U.S.A.
}

(Received 10 December, 1971; in revised form 8 February, 1972)

\begin{abstract}
Peak fluxes of flare-associated 8-12 $\AA$ X-ray bursts occur at or near the time of the maximum energy content of the soft X-ray source volume. The amplitudes of flare-associated bursts may thus be used as a measure of the energy deposited in the source volume by non-thermal electrons and other processes. In the mean, the soft X-ray burst amplitude is apparently independent of the occurrence of a type III event. This is interpreted to indicate that electrons accelerated by the type III process do not directly participate in establishing the soft X-ray source volume.
\end{abstract}

\section{Introduction}

Considerable attention has been directed towards the so-called flash phase (Ellison, 1949) or explosive phase (Athay and Moreton, 1961; Harvey, 1971) of solar flares, because it is thought that during this phase electrons are rapidly accelerated to high energies in the solar atmosphere. A number of flare models have been proposed (e.g., Arnoldy et al., 1968; Takakura, 1969; Holt and Ramaty, 1969; Parks and Winckler, 1971) that explore in particular the possibility that hard $X$-rays and $\mathrm{cm}-\lambda$ radiation may both be generated by non-thermal electrons that result from the acceleration process.

An important question is whether the non-thermal electrons represent a significant energy supply to other associated aspects of the flare phenomenon. Neupert (1968) and Kahler et al. (1970) proposed that fast electrons generated by an initial acceleration event collisionally heat the thermal X-ray source volume in addition to producing non-thermal radiation. Kane and Donnelly (1971) extended the concept further when they deduced that the total kinetic energy of fast electrons is comparable to the energy that is deposited in the source volume which gives rise to the EUV flash, while Lin and Hudson (1971) have inferred that as much as one-half of the total flare energy is initially represented by the energy of non-thermal electrons. Thus recent observationally-based studies strongly suggest that non-thermal electrons are indeed an important energy supply for the flare event.

In addition to the impulsive onset of flare bursts, by which is deduced the operation 
of an acceleration mechanism, the occurrence of meterwave type III events also signifies an acceleration process, undoubtedly connected with and perhaps identical to the process which provides the electrons for the microwave burst. The type III radiation is considered to be connected with the acceleration of $\gtrsim 10^{35}$ electrons to energies of tens of $\mathrm{keV}$ (Wild, 1964). Lin and Hudson found that in two flares accompanied by type III radiation at X-ray maximum $\left(E_{e}>22 \mathrm{keV}\right)$, the number of electrons escaping from the Sun was greater than in two flares not accompanied by type III. They calculated (their Table II) that the total number of electrons of energy exceeding $22 \mathrm{keV}$ and the aggregate electron energy tended to be greater for the flares accompanied by type III.

If we adopt the point of view that the type III process supplies additional electrons to those provided by the mechanism that gives rise to the impulsive microwave burst, then possibly some of these additional electrons might contribute to the total energy of the thermal X-ray source, depending upon how the electrons propagate out of the volume in which they are accelerated. If some of the type III electrons propagate downwards towards the chromosphere, we would expect to observe an enhancement in the energy content of the termal X-ray volume when flares accompanied by type III are compared with flares not associated with type III.

We have tested this idea using soft X-ray data between 8 and 12 angströms acquired by an ion chamber photometer on board OSO 3. Our results are negative and indicate that the soft X-ray source volume is essentially similar in flares whether or not the meter-wave type III event is detected.

In Section 2 we describe our approach to the problem and discuss its validity. Section 3 presents the results.

\section{Data Selection and Analysis}

A catalog of distinct, non-overlapping $\mathrm{H} \alpha$ flare events for which OSO 3 data were available was prepared by Thomas (1970) for the period 10 March 1967, to 31 March 1968. Flare events studied here have been drawn from this catalog (Catalog II) which was briefly described by Thomas and Teske (1971).

Only flares of importance 1 which met certain criteria were analyzed for the project reported here. Our method of analysis was straightforward: the sample of flares was divided into groups according to whether a type III burst did or did not occur, and mean soft $X$-ray amplitudes obtained for each grouping of events. These means were then tested against one another to determine if differences in group means were statistically significant. This approach to the data requires some explanation and some justification.

\section{A. SELECTION CRITERIA}

Our intent is to demonstrate to what extent the energetic electrons associated with occurrence of the type III acceleration process participate in establishing the soft $\mathrm{X}$-ray source in flares. 
As we examine flares of greater and greater importance, we find that the peak soft X-ray and $\mathrm{H} \alpha$ fluxes increase in the mean (Thomas and Teske, 1971) and that the frequency of association with bursts of type III also increases (Swarup et al., 1960). However, this may only indicate that the more energetic phenomena are better capable of producing fast-drift bursts. To avoid this possible bias we have elected to examine only flares of importance 1 .

In the mean, the total $\mathrm{H} \alpha$ emission from a flare is roughly proportional to the total soft X-ray emission (Thomas and Teske, 1971). Since flares of importance 1 will share a common range of total $\mathrm{H} \alpha$ emission, the present study thus has attempted to distinguish whether or not a greater proportion of soft X-radiation accompanies the $\mathrm{H} \alpha$ aspect in those events associated with type III.

Only those flares of importance 1 in Catalog II were analyzed which occurred during hours when a swept-frequency station was observing. (Because we were not familiar with the reports from Weissenau, we did not include them in our data if that was the only reporting station.) Only those flares between solar longitudes $75^{\circ} \mathrm{E}-30^{\circ} \mathrm{W}$ were examined for the type III/soft X-ray association. Loughead et al. (1957) showed that the highest visibility of type III occurs in the solar longitude range $90^{\circ} \mathrm{E}-30^{\circ} \mathrm{W}$. We eliminated importance 1 flares between $90^{\circ} \mathrm{E}-75^{\circ} \mathrm{E}$ in this study so as to avoid the inevitable problems of flare importance assignments that arise close to the limb (e.g., Sawyer, 1967). All flares for which an associated type II and/or type IV was observed were excluded so as to isolate only the type III acceleration mechanism (or its absence).

\section{B. USE OF SOFT X-RAY BURST AMPLITUDES}

We have used our observed 8 to $12 \AA$ burst amplitudes as a measure of energy deposited in the source volume. This must first be justified.

(i) It has been shown (Teske et al., 1971) that the Michigan OSO 3 ion chamber detector is relatively insensitive to plasma temperature $T$ in the range $10 \times 10^{6} \mathrm{~K}$ $20 \times 10^{6} \mathrm{~K}$ : the measured ion chamber current produced by a fixed emission measure $N_{e}^{2} \mathrm{~V}$ will vary by about $50 \%$ across this range of temperature. This conclusion was based upon calculations which took account of free-free, free-bound and line emissions.

Horan (1970) has studied the time-histories of emission measure and plasma temperature in 17 flares using observations made with $0.5-3.0 \AA$ and $1-8 \AA$ ion chambers on OGO 4. A comparison of his results with our OSO 3 data indicates that at the peak of the 8-12 $\AA$ burst, the equivalent temperature of the $0.5-8 \AA$ source, as deduced by him, is in the range $13 \times 10^{6} \mathrm{~K}-18 \times 10^{6} \mathrm{~K}$. Horan compared the temperatures derived from the OGO 4 data with temperatures deduced from continua observed with a Bragg crystal spectrometer on OSO 4 (Meekins et al., 1970), finding that the OGO ion chamber data led to temperatures $15 \%-30 \%$ below the Bragg crystal results. This occurred because only free-free and free-bound continua were considered in analysing the ion chamber observations; line emission was neglected. However, Horan's results indicate that plasma temperatures appropriate to the $8-12 \AA$ source being 
observed by us probably indeed lie in the range $10 \times 10^{6} \mathrm{~K}-20 \times 10^{6} \mathrm{~K}$ at the $8-12 \AA$ peak, at temperatures where our detector responds principally to variations in emission measure.

We assume that the thermal soft X-ray source volume in a 'mean' importance 1 flare is characterized by a 'mean' emission measure and a 'mean' temperature. Since our instrument is insensitive to temperature, significant differences in mean soft X-ray amplitude between various sample groups of flares may be taken to indicate variations in mean emission measure.

Kahler et al. (1970) pointed out that the total thermal energy content of the soft $\mathrm{X}$-ray source may be approximated by

$$
E \sim 2.6\left(N_{e}^{2} V\right)^{1 / 2} V^{1 / 2} k T
$$

where $V$ is the equivalent volume of the source and the factor 2.6 takes into account the solar chemical abundances. Although our peak burst fluxes may be used to specify differences in mean emission measure according to the arguments above, these would not themselves indicate mean differences in total thermal energy content unless we also have information on $T$ and on $V$.

To anticipate our results: we have found no evidence for differences in mean 8-12 $\AA$ burst amplitude exceeding a factor of 1.7 among the sample flare groups studied. Statistical discussion of the results suggests that any differences are in fact less than this value. Assuming that there is a mean temperature which characterizes the 8-12 $\AA$ source in a mean flare of importance 1 , this indicates that the mean emission measure for these sample groups is the same within a factor of 1.7 . It would be quite fortuitous for $N_{e}$ and $V$ to vary separately within individual flares in such a way as to produce this result, hence on the average $N_{e}^{2}$ and $V$ both are the same to within a factor of less than two in, for example, a mean flare of importance $1 \mathrm{n}$, whether or not a type III burst accompanied it. We thus infer that the mean total energy content of the source at the time of burst peak is the same, within a factor of less than two.

(ii) Horan's time-histories of $T$ and $N_{e}^{2} V$ in flares may be used with our Equation (1) to show that the thermal energy content of the $0.5-8 \AA$ source region is near its maximum at the time of the $8-12 \AA$ burst peak, under the assumption that $V^{1 / 2}$ varies only slowly with the time. We were able to compare 8 of Horan's 17 events with our own OSO 3 data. In six of the eight flares, our OSO $38-12 \AA$ burst peak coincided within one minute of the time of maximum total energy of the $0.5-8 \AA$ source, while in the other two flares the total energy in that source reached a maximum slightly earlier than the $8-12 \AA$ peak ( $4 \mathrm{~min}$ and $2.5 \mathrm{~min}$, respectively).

Horan demonstrated that temperatures derived from the OGO data were $15 \%-30 \%$ below those deduced from the OSO Bragg crystal data, but the errors (obtained from his Table 5-2) appear to be nearly temperature-independent across the range $9 \times 10^{6} \mathrm{~K}-17 \times 10^{6} \mathrm{~K}$. In his analysis, both free-free and free-bound continua were calculated for coronal chemical abundances using, for the free-free emission, a 'representative' value of the Gaunt factor for each ion chamber wavelength band. The free-bound emission was evaluated with a slightly different expression than that 
used by Culhane (1969). Thus, values of emission measure obtained by Horan are about $30 \%$ smaller than would be obtained had Culhane's continua been employed, although the plasma temperature determination is unaffected by the difference, as Horan pointed out. The temperature dependence of the ratio of free-bound to total continuum flux is in agreement with Culhane's, as well. There is, therefore, an uncertainty in the time-history of the relative total energy content of the $0.5-8 \AA$ source as deduced from Horan's results, and a corresponding uncertainty in the above comparison which indicates good coincidence between the time of the 8-12 $\AA$ burst peak and the time of maximum energy content of the $0.5-8 \AA$ source. Because of the weak temperature dependence of possible errors in Horan's results, however, we do not expect that the relative times of these phenomena would change significantly if the errors were taken fully into account.

(iii) Kahler et al. (1970) have deduced that the emission measure of the $5-10 \mathrm{keV}$ (1.2-2.4 $\AA$ ) X-ray source increases after the X-ray peak, that the temperature slowly declines and that the thermal energy content of the gas remains nearly constant over an extended period of time. If this latter deduction is correct, our use of the $8-12 \AA$ burst amplitudes as a measure of the energy deposited in the source volume is questionable.

In their analyses of the 9 June 1968 and 9 July 1968 events, Kahler et al. determined temperatures from spectrum fits of free-free bremsstrahlung curves between 5 and $10 \mathrm{keV}$ photon energies, and emission measures from the derived $T$ and a flux in one photon energy channel. While line emission at $1.9 \AA$ was accounted for, they ignored the effects of free-bound emission. As they pointed out, that omission had little effect upon the derived temperature, which declined during the flares, although the emission measure was overestimated. The overestimate is temperature-dependent. For example at a temperature of $12.5 \times 10^{6} \mathrm{~K}$, the emission measure determined by them was overestimated by a factor of about 1.7 , while at $10 \times 10^{6} \mathrm{~K}$, the overestimate is by a factor of about 2.0. Using the data published by Kahler et al. we have reanalyzed the 9 June 1968 event, accepting their temperatures but determining emission measures by taking into account both free-free and free-bound continua. We find that although the emission measure $N_{e}^{2} V$ did rise a bit near the X-ray maximum, the thermal energy content of the source declined after $08^{\mathrm{h}} 55^{\mathrm{m}}$. Furthermore, at $09^{\mathrm{h}} 01^{\mathrm{m}}$, the time of the 2-12 $\AA$ peak flux observed from Explorer 35, the thermal energy content was declining more rapidly than had been suggested by the original analysis.

The flare plasma is undoubtedly highly complicated thermodynamically, spatially and temporally. It is not immediately clear what relationship the 8-12 $\AA$ source observed by us has with the $0.5-8 \AA$ source observed by Horan, or with the $5-10 \mathrm{keV}$ source observed by Kahler et al. If we adopt the usual viewpoint that the bulk of radiation at shorter $\mathrm{X}$-ray wavelengths is emitted by hotter parts of a continuum of flare plasma, with the bulk of the longer wavelength emission coming from cooler and perhaps more extensive parts of the plasma, we may assume that those source regions to which Horan's data refer are at higher temperatures than is the $8-12 \AA$ source region, while the data of Kahler et al. would refer to still hotter gas. However, both analysis revealed 
source temperatures in the range of $10-20 \times 10^{6} \mathrm{~K}$, and both demonstrated that the source temperature was declining only slowly at the time of the $8-12 \AA$ or $2-12 \AA$ burst peak. Since our OSO 3 detector is insensitive to temperature in this range, the time of peak flux will closely indicate the time of peak emission measure in the 8-12 $\AA$ source region. Assuming that in the 8-12 $\AA$ source region the temperature also slowly declines near the burst peak, we conclude that the total energy in the 8-12 $\AA$ source is also near its maximum at the time of the 8-12 $\AA$ burst maximum, and that the total energy declines thereafter. This conclusion is consistent with the observations discussed above, which show that the total energy content of the $5-10 \mathrm{keV}$ source and of the $0.5-8 \AA$ source also decline after the $8-12 \AA$ burst peak.

The $8-12 \AA$ amplitude, while measuring the energy content of the soft X-ray source at the time of burst maximum, does not, however, provide a measure of the total energy which is deposited during the entire flare event.

\section{Results}

\section{A. TYPE III BURSTS}

The sample of importance 1 flares in Catalog II which occurred during swept-frequency station observing hours and between solar longitudes $75^{\circ} \mathrm{E}-30^{\circ} \mathrm{W}$ was divided into two groups, depending upon whether a Type III burst was associated with the flares. For many flares only partial data were available. Mean 8-12 $\AA$ amplitudes were calculated for each sample group. The means are compared in Table $I$. The errors which are given are errors of the mean (m.e.). In the table, $P$ is a parameter, calculated by Student's $t$-test, expressing the significance of the difference between the sample means. (Energies quoted in Table I have not been corrected via the correction factors suggested by Teske et al., 1972.)

However, the data in Table I do not take account of possible accidental associations with a type III burst which arises from another active center fortuitously at the time of a flare. If $N_{0}=$ the real number of flares without type III and $N_{\text {III }}=$ the real number of flares with Type III, the observed quantities are $N$ and $N_{\text {III }}^{\prime}$ :

$$
\left.\begin{array}{c}
N=N_{0}+N_{\text {III }} \\
N_{\text {III }}^{\prime}=N_{\text {III }}+k N_{0}
\end{array}\right\}\left\{\begin{array}{c}
N_{0}=\frac{N-N_{\text {II }}^{\prime}}{1-k} \\
N_{\text {III }}=\frac{N_{\text {III }}^{\prime}-k N}{1-k}
\end{array}\right.
$$

where $k$ is the probability of accidental association. Assuming that the mean soft X-ray burst amplitude for non-type III-associated flares, $E_{0}$, has been correctly deduced, the true burst amplitude for Type III-associated flares is

$$
E_{\mathrm{III}}=\frac{(1-k) N_{\mathrm{III}}^{\prime} E_{\mathrm{III}}^{\prime}-\left(N-N_{\mathrm{III}}^{\prime}\right) k E_{0}}{N_{\mathrm{III}}^{\prime}-k N}
$$

where $E_{\mathrm{III}}^{\prime}$ has been obtained from measures which include spurious Type-III associa- 


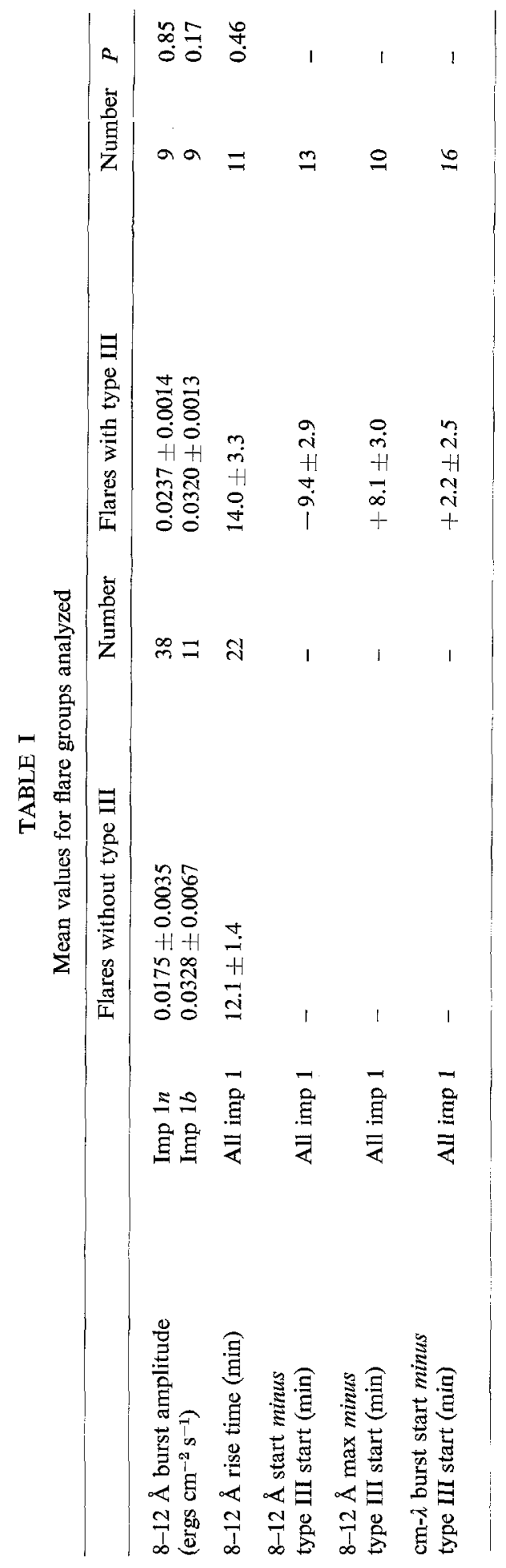


tions. $k$ may be ascertained from the observed frequency of occurrence of type III and from the association interval $\Delta t: 12 \mathrm{~min}$ was used in this study. Since the daily rate of type III occurrence is highly variable and the data used here spanned nearly ten months, we have not computed $k$ in this way. Swarup et al. (1960) found that about $20 \%-24 \%$ of flares are in reality accompanied by type III. We have found (Table I) $18 / 67=27 \%$, without correcting for chance associations.

To examine the possible effects on our results of chance associations, we assumed that half of the associated events were spurious, i.e. $N_{\mathrm{III}}=0.5 N_{\mathrm{III}}^{\prime}$, and computed $k$ and $E_{\mathrm{III}}$ under this assumption. In that case, the mean soft X-ray burst amplitude for importance $1 \mathrm{n}$ flares with type III rises to $0.0299 \mathrm{ergs} \mathrm{cm}^{-2} \mathrm{~s}^{-1}$ (1.7 larger than for importance $1 \mathrm{n}$ flares without type III), while that for the importance $1 \mathrm{~b}$ flares rises to $0.0322 \mathrm{ergs} \mathrm{cm}^{-2} \mathrm{~s}^{-1}$. Since we cannot identify the spurious events, we assumed that the mean errors in those values remained the same as before and thus computed that the parameter $P$ for importance 1 f flares increases to $0.96\left(4^{\%}\right.$ probability that the amplitude difference is due to chance) while $P$ for the $1 \mathrm{~b}$ flares is not significantly changed.

There is no obvious physical reason which might explain why importance $1 \mathrm{n}$ flares with type III should have greater soft X-ray bursts than those without type III while at the same time the burst amplitudes with importance $1 \mathrm{~b}$ flares are independent of type III, as these statistics suggest. Thus the correction for spurious associations does not lead to a clear-cut demonstration that there is any real physical difference.

Although there is no compelling statistical evidence to favor a real difference in mean 8-12 $\AA$ burst amplitude of importance $1 \mathrm{n}$ and $1 \mathrm{~b}$ flares with and without type III, a possible difference of a factor of 1.7 exists for the importance 1 n flares. According to our arguments of Section $2 b(i)$, we interpret this to indicate that the total energy of the thermal soft X-ray source in importance 1n flares accompanied by type III is not more than a factor of two greater than in importance 1 n flares not accompanied by type III.

On the average (Table I), the type III burst begins well before the soft X-ray maximum (in only one instance did it occur afterwards) and at a time considerably after the commencement of the soft X-ray burst, although a wide scatter exists. There is no detectable relationship between occurrence of a type III and the impulsiveness of the soft $\mathrm{X}$-rays as revealed by rise times (Table I), even though the type III, when it occurs, preferably commences on the rising branch of the X-ray curve.

On the other hand, from the same sample of flares we find a tendency for the microwave burst to commence shortly after the type III burst (see Table I), thus suggesting a connection between the accelerated electrons (or the acceleration events) which give rise to the two kinds of bursts, but this tendency is not clearly established by the data.

\section{B. DISCUSSION}

These results are entirely consistent with an earlier examination of the association 
of type III bursts with soft X-rays in the absence of flares (Teske et al., 1971) in which it was found that only a weak soft $\mathrm{X}$-ray rise occasionally accompanies a non-flare type III, the X-rays beginning somewhat earlier than the fast-drift event. The present extension of that work to flares suggests that the visible fast-drift phenomenon has no significant influence upon the amplitude of the 8-12 $\AA$ X-ray burst in flares which which are classified importance $1 \mathrm{n}$ or $1 \mathrm{~b}$.

The observational basis for models in which non-thermal electrons heat the X-ray source has been confined to photon energies $E_{h v} \gtrsim 3 \mathrm{keV}$ and to the EUV region. Because the flux/time curves at photon energies $E_{h v} \simeq 1-1.5 \mathrm{keV}$ used here are so different from those at the higher photon energies, it is questionable whether we may view our data entirely in terms of these models. A second reason for questioning whether the $1-1.5 \mathrm{keV}$ data may be viewed in the context of a collisional-heating model is that the soft X-rays tend to precede both the $\mathrm{cm}-\lambda$ and the type III bursts (Teske and Thomas, 1969; Culhane and Phillips, 1970). Thus at least a part of the $1-1.5 \mathrm{keV}$ source may not be the outcome of precipitation of non-thermal electrons.

Evidence in favor of assuming the $8-12 \AA$ source to be mainly heated by electron precipitation from the microwave source lies in the relationship between amplitudes of the soft X-ray burst and the cm- $\lambda$ burst, and between the time-integrated energies in soft X-rays and $\mathrm{cm}-\lambda$ (Teske, 1969), relationships that are more firmly established by a recent assessment of the energy scale of our ion-chamber detector (Teske et al., 1971). Further, the $8-12 \AA$ peak flux follows the $\mathrm{cm}-\lambda$ burst peak, as we would expect from the collisional-heating model.

These energetic and temporal relationships appear to us to indicate that while a significant part of the $8-12 \AA$ burst source is established by electron precipitation, a minor part of the source may be established by other mechanisms. Our present work indicates that the type III acceleration process, when it occurs, does not lead to an appreciably stronger soft X-ray burst in flares of importance 1 .

\section{Summary}

The work of other investigators cited above indicates that the thermal soft X-ray source volume in solar flares is, at least in part, collisionally heated by non-thermal electrons associated with the impulsive microwave burst. We have previously shown that the $8-12 \AA$ emission from flares is quantitatively associated with the microwave emission, and infer that an important fraction of the X-ray source being observed at these wavelengths is also the result of heating by electrons connected with the microwave emission. The time of peak burst flux in the 8-12 $\AA$ band is near or at the time of maximum thermal energy content of the soft X-ray source. The work reported here indicates that electrons associated with a type III event observed at meter wavelengths do not lead to an appreciably stronger soft X-ray burst in flares of importance 1 than would usually be found in importance 1 flares in the absence of a type III burst. 


\section{Acknowledgements}

It is a pleasure to thank Dr Z. Švestka and an anonymous referee for their comments on a manuscript of this paper. The work was supported in part under NASA Grant NGR-23-005-367.

\section{References}

Arnoldy, R. L., Kane, S. R., and Winckler, J. R.: 1968, Astrophys. J. 151, 711.

Athay, R. G. and Moreton, G. E.: 1961, Astrophys. J. 133, 935.

Culhane, J. L.: 1969, Monthly Notices Roy. Astron. Soc. 144, 375.

Culhane, J. L. and Phillips, K. J. H.: 1970, Solar Phys. 11, 117.

Ellison, M. A.: 1949, Monthly Notices Roy. Astron. Soc. 109, 3.

Harvey, K. L.: 1971, Solar Phys. 16, 423.

Holt, S. S. and Ramaty, R.: 1969, Solar Phys. 8, 119.

Horan, D. M.: 1970, Dissertation, Catholic University of America.

Kahler, S. W., Meekins, J. F., Kreplin, R. W., and Bowyer, C. S.: 1970, Astrophys. J. 162, 293.

Kane, S. R. and Donnelly, R. F.: 1971, Astrophys. J. 164, 151.

Lin, R. P. and Hudson, H. S.: 1971, Solar Phys. 17, 412.

Loughead, R. E., Roberts, J. A., and McCabe, M. K.: 1957, Australian J. Phys. 10, 483.

Meekins, J. F., Doschek, G. A., Friedman, H., Chubb, T. A., and Kreplin, R. W.: 1970, Solar Phys. 13, 198 .

Neupert, W. M.: 1968, Astrophys. J. (Letters) 153, L59.

Parks, G. K. and Winckler, J. R.: 1971, Solar Phys. 16, 186.

Sawyer, C.: 1967, Astrophys. J. 147, 1135.

Swarup, G., Stone, P. H., and Maxwell, A.: 1960, Astrophys. J. 131, 725.

Takakura, T.: 1969, Solar Phys. 6, 133.

Teske, R. G.: 1969, Solar Phys. 6, 193.

Teske, R. G., Soyumer, T., and Hudson, H. S.: 1971, Astrophys. J. 165, 615.

Teske, R. G., Hodge, P. E., and Worden, S. P.: 1971, Solar Phys. 22, 235.

Thomas, R. J.: 1970, Dissertation, University of Michigan.

Thomas, R. J. and Teske, R. G.: 1971, Solar Phys. 16, 431.

Wild, J. P.: 1964, Symposium on the Physics of Solar Flares, p. 161, NASA SP-50. 